\title{
An Image Segmentation Method for Early Detection and Analysis of Melanoma
}

\author{
Arushi Bhardwaj ${ }^{1}$, Dr. J.S Bhatia ${ }^{2}$ \\ Centre for Development of Advanced Computing, Mohali, India, 160071
}

\begin{abstract}
Skin cancer through all types of cancers, is the least frequent and most common form of human cancers. Skin cancer may be Melanoma, Basal and Squamous cell among which Melanoma is the most precarious. It is compulsory to recognize it in its early stage for its proper cure. In this paper, image segmentation method is implemented in MATLAB followed by STOLZ and TDS algorithm. An image segmentation method is used to classifying the current state of melanoma lesions. It further includes various steps for analysis; preprocessing which is used for image enhancement, removal of noise, hair. Then the boundary of the skin lesion is detected with the help of initial segmentation. The lesion is investigated to find the parameters using feature extraction property. The fetched parameter values are therefore used to calculate STOLZ algorithm and by feeding the output of the STOLZ algorithm in TDS, Detection of the current stage of melanoma can be identify.
\end{abstract}

Keywords: Image segmentation, Feature extraction, STOLZ algorithm, TDS.

\section{INTRODUCTION}

The Skin biology is a vast field. Body's largest organ is Skin. It saves us from various infections, sunlight and from many other problems and helps us to maintain body temperature [1]. As, Skin itself is a very complicated structure consists of different types of tissues. These tissues can go through cancerous conversion during their development process resulting in cancer with different biological attitude. Cancer is defined as disease which is caused due to abnormal growth of cells and covers others body parts with the help of blood and lymph system. The expansion of the cancer tissues is very much different from normal cell expansion. The important factor is rather than vanishing the cancer cells it increase continuously and produce abnormal cells. The DNA cause cell to become cancer cells [3].Figure 1.1 differentiates cancer cell growth from normal growth of cell .Above 100 types of cancer are possible on the skin which are clinically proven and are having genetic components with many other properties. Melanoma is a type of Skin Cancer and it originates in Melanocytes [1]. It can be in any part of the body and can hit anyone. Every year, approximately 55,000 peoples found suffering from this kind of cancer. As compared to all other types of cancer, Melanoma is quite common.

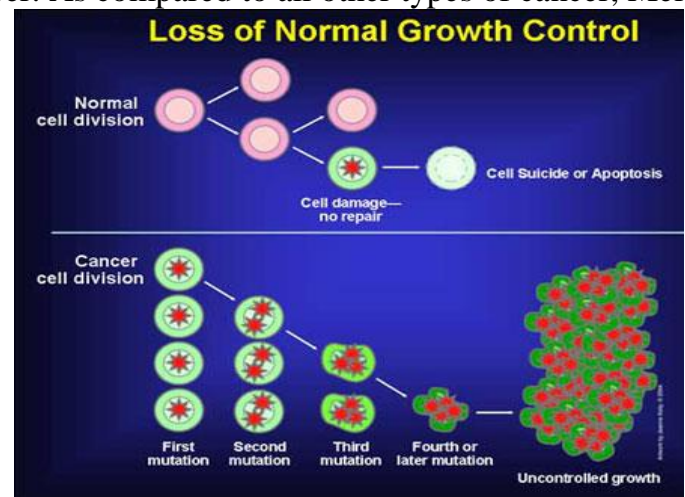

Figure 1.1: Normal and Cancer cell growth

\section{Cancer Directory}

Epidermis: - Epidermis is top most layer of the skin. It is made of dead cells and contains Kerotinocytes and Melocytes [1].

Dermis: - It is the lower layer of the skin contains fats. The main function of this layer is to feed, excrete and exchange of heat. In this layer, Fat cells act as the most important cells [1].

Incidence: - Incidence rate of cancer is the division of number of new cases to total number of persons multiplied by 100,000 .Numerical representation is (same type of cancer is considered at one time) [4].

I R $=$ (New cases/Total persons) X 100,000. 
Mortality: - Mortality rate of cancer division of no. of deaths cases to the total no. of persons multiplied by 100,000 .Numerical representation is (same type of cancer is considered at one time) [4].

$$
\text { M R = (Cancer Deaths/Total persons) X 100,000. }
$$

Prevalence: - Number of live person on specific date who is already being diagnosed of the disease. It contains the new and already existing cases. This factor is very useful for the planning of health and resource [4].

Survival: - Survival rate is basically expressed as the ratio of a live patient to the analysis of tumor. Relative survival is a percentage estimate of the patients who are expected to survive Observed survival is the actual percentage of patient's still alive after examination [4].

Lifetime Risk: - It is defined as the maximum chances of growing of tumor. It also helps in the calculation of the certain age wise development of tumor [4].

\section{Design Flow}

By considering figure 1.2 the flow chart is mainly shown in two main blocks i.e. Image analysis block which contain image segmentation procedure and second is melanoma detection block which consist of STOLZ algorithm and TDS parameter value that gives detection result. For early detection of melanoma, the very first and basic requirement of the diagnosis is the acquisition of digital images of melanoma. Melanoma images of the patients are of different pixels and sizes. In actual practice these images contains various kind of noise, and other particles like hair, bubbles etc. and for the removal of this noise image segmentation is performed.

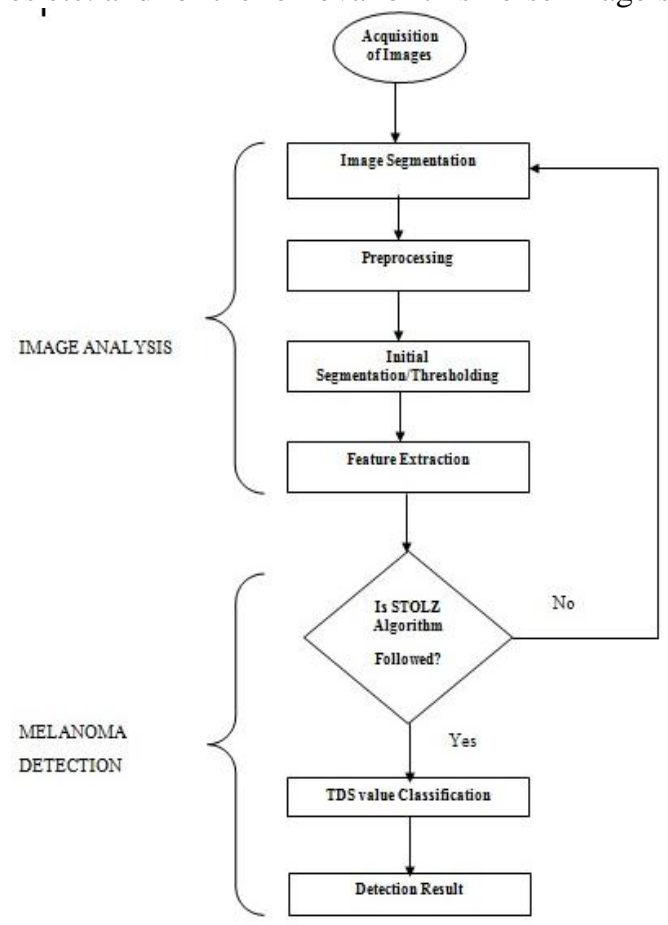

Figure 1.2: Design flow of proposed method

The constructive view of proposed method is shown in figure 1.3

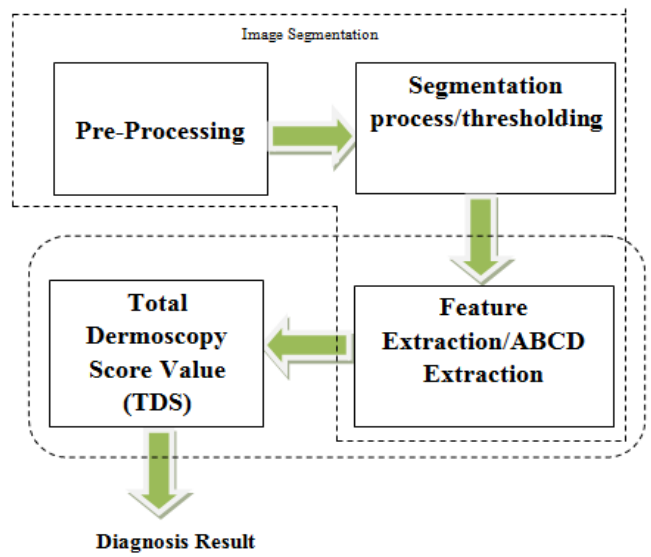

Figure 1.3: Constructive view of proposed method 


\section{Results}

The software tool used is MATLAB R2013a (8.1.0.604) version. The original image is in jpeg format \& having RGB color model which is then converted to grayscale image.

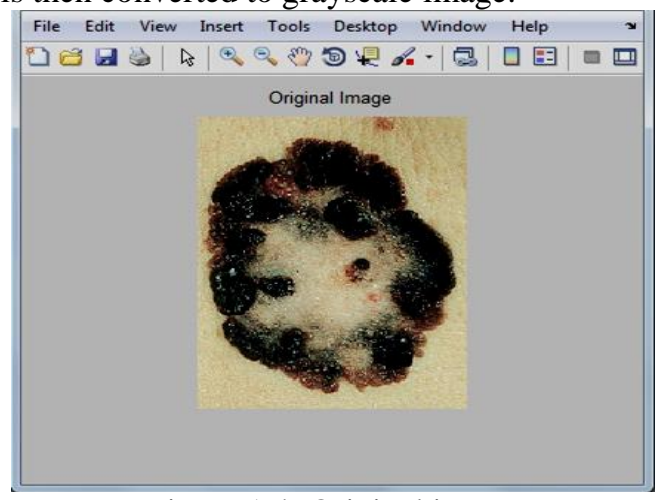

Figure 1.4: Original image

In order to fetch the features of the image, regionprops command is used. The need of area, perimeter and Minor axis length is to calculate the asymmetry, border irregularity and diameter of the mole.

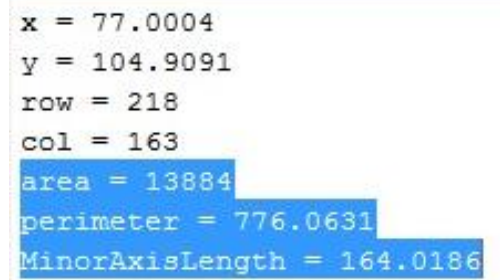

After the extraction of the mole features the next is the implementation of STOLZ algorithm. If the mole is asymmetrical, then these two bisected half are not equal to each other as shown in the figure 1.5

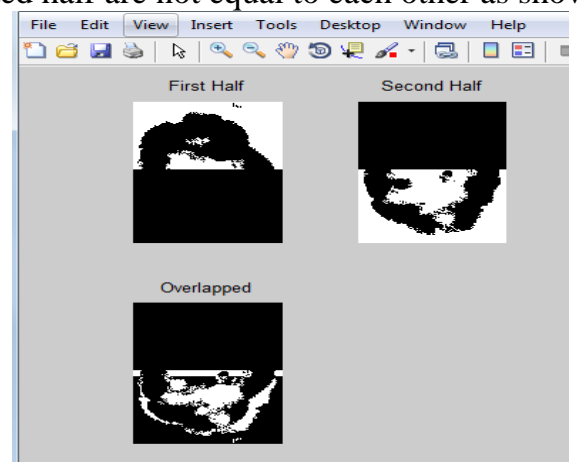

Figure 1.5: Bisection of the image in upper and lower part with an overlap image to get asymmetry. The mathematical expression used to calculate asymmetry is:-

Where,

$$
\text { Asymmetry }=(\Delta \mathrm{T} / \mathrm{T}) * 10
$$

$\Delta \mathrm{T}=$ Pixel difference

$\mathrm{T}=$ Total Pixel count of lesion

$\mathrm{A}=0.73233$ (approx) pixel or $0.194 \mathrm{~mm}$

Border : - The border is irregular in case of Melanoma. So, the edge or the border are first recognized and then fetched from the image excluding the inner, outer parts of the mole

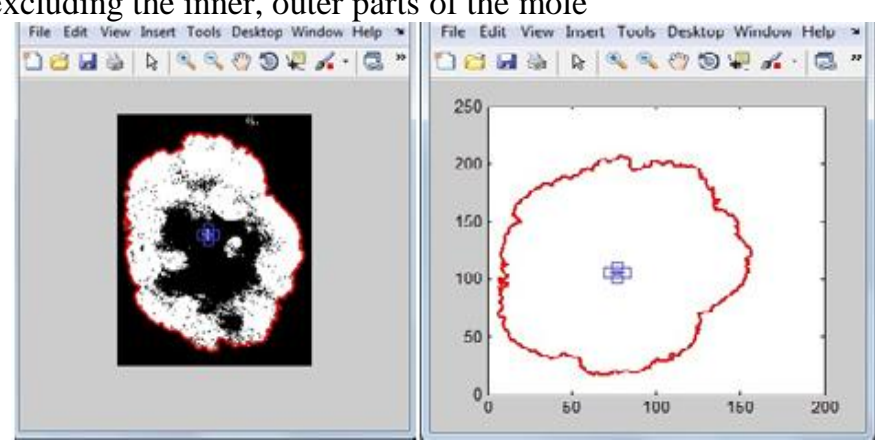

Figure 1.6: Edge and border detection of an image 
The border irregularity is calculated using

Where, $A=$ Area of the lesion

$$
\mathrm{B}=\left[(\text { perimeter })^{2} / 4 \pi \mathrm{A}\right]
$$

$$
\pi=22.7
$$

After calculation the border irregularity value is $B=0.47774$ pixel or $B=0.13 \mathrm{~mm}$

Color :- The third dimension of the skin mole is color. If the mole is having more than one color

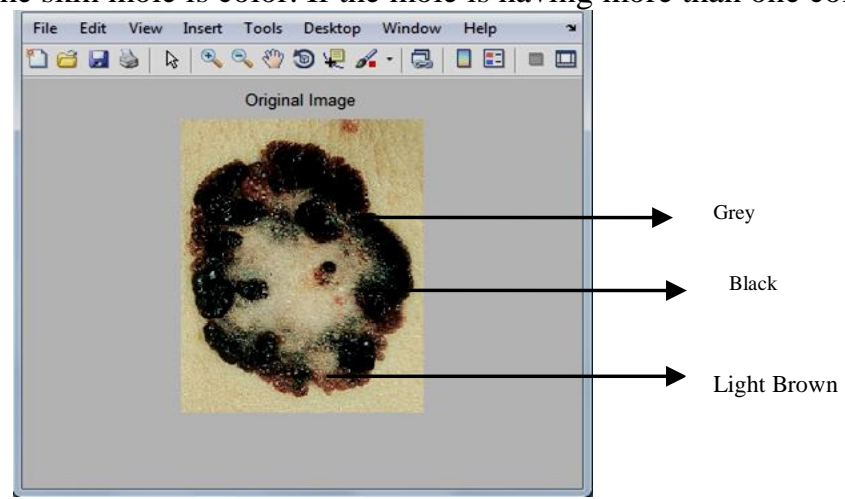

Figure 1.7: Colors of Skin mole

Dermoscopic structure it contains one or more than one structure inside itself. Dermoscopic structure $=2$ structures.

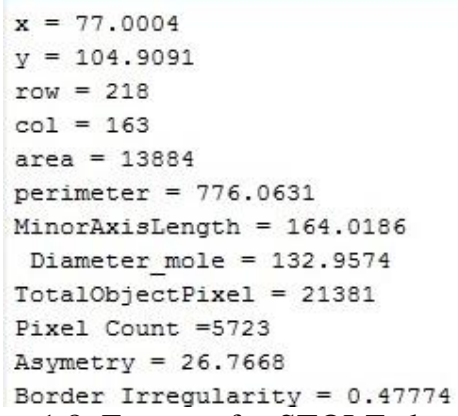

Figure 1.8: Features for STOLZ algorithm

Here multiply factor for $\mathrm{ABCD}$ are as follows

$\mathrm{A}=0.72 * 1.3$

$\mathrm{B}=0.13 * 0.1$

$\mathrm{C}=3 * 0.5$

$\mathrm{D}=2 * 0.5$

Table1: Weight multiply factor

\begin{tabular}{|l|c|c|}
\hline CRITERION & SCORE & $\begin{array}{c}\text { MULTIPLY } \\
\text { FACTOR }\end{array}$ \\
\hline Asymmtrey & $0-2$ & 1.3 \\
\hline Border & $0-8$ & 0.1 \\
\hline Color & $1-6$ & 0.5 \\
\hline $\begin{array}{l}\text { Dermoscopic } \\
\text { Structure }\end{array}$ & $1-5$ & 0.5 \\
\hline
\end{tabular}

$\mathrm{TDS}=\left[\left(\mathrm{A}^{*} 1.3\right)+(\mathrm{B} * 0.1)+(\mathrm{C} * 0.5)+(\mathrm{D} * 0.5)\right]$----------- [Equation 1]

TDS = 3.44 (using Equation 1)

For final diagnosis result, classification is done using TDS table (refer table 1)

By getting the TDS value the result comes out is benign skin lesion. It is an early stage of Melanoma

TDS is $<4.75$ i.e. 3.44 therefore it is

\section{Benign Skin Lesion}

\section{Conclusion}

In this paper, Melanoma image is processed using image segmentation method in MATLAB and then the features of the same is implemented on STOLZ Algorithm and verified according to TDS parameter in order to analysis the stage of melanoma, here the Melanoma is in Benign stage so that it can be cure in early stage. 


\section{References}

[1] D J Gawkrodger,Dermatology an illustrated colour text. Elsevier health science, 2003.

[2] Chudnovsky , Yakov,Paul A.Khavari, and Amy E. Adams."Melanoma genetics and the development of rational therapeutics." Journal of clinical Investigation 115.4 (2005): 813-824

[3] National Cancer Institute, "Surveillance, Epidemiology, and End Result program," http://seer.cancer.gov/statfacts/html/melan.html, April 15, 2014.

[4] “American Cancer Society,” http://www.cancer.org/cancer/skincancer/index, Oct 10, 2013.

[5] “Cancer council Victoria,”http://www.cacervic.org.au/about-cancer/cancer_types/melanoma, Oct 31, 2012.

[6] Ignazio Stanganelli, MD Skin Cancer Unit, IRST, Meldola, and Ospedale Niguarda, Milan, Italy,“ Melanoma Molecular Map Project ," http://www.mmmp.org/MMMP/import.mmmp?page=dermoscopy.mmmp , Oct , 2008

[7] Madhankumar, K., and P. Kumar, "Characterization of skin lesions", Pattern Recognition, Informatics and Medical Engineering (PRIME), 2012 International Conference on. IEEE, 2012.

[8] Saini, Harpreet Kaur, and Onkar Ch. "Skin Segmentation Using RGB Color Model and Implementation of Switching Conditions." Skin 3.1 (2013): 1781-1787.

[9] Silveira, Margarida, et al. "Comparison of segmentation methods for melanoma diagnosis in dermoscopy images." Selected Topics in Signal Processing, IEEE Journal of 3.1, pp. 35-45, 2000.

[10] Pham, Dzung L., Chenyang Xu, and Jerry L. Prince. "Current methods in medical image segmentation 1." Annual review of biomedical engineering 2.1, pp. 315-337, 2000.

[11] Abercrombie, M., and E. J. Ambrose. "The surface properties of cancer cells: a review." Cancer research 22.5 Part 1, pp. 525-548, 2003.

[12] Garnavi, Rahil, Mohammad Aldeen, and James Bailey. "Computer-aided diagnosis of melanoma using border-and wavelet-based texture analysis.” Information Technology in Biomedicine, IEEE Transactions on 16.6, pp. 1239-1252, 2012.

[13] Kosovsky, Marguerite. "Cancer stem cell research.”,pp. 1-8, 2012.

[14] Narayanan, Deevya L., Rao N. Saladi, and Joshua L. Fox. "Review: Ultraviolet radiation and skin cancer.” International journal of dermatology 49.9 (2010): 978-986.

[15] Blum, A., et al. "Digital image analysis for diagnosis of cutaneous melanoma. Development of a highly effective computer algorithm based on analysis of 837 melanocytic lesions.” British Journal of Dermatology 151.5 (2004): 1029-1038.

[16] Xu, Jin Wei, Tuan D. Pham, and Xiaobo Zhou. "A double thresholding method for cancer stems cell detection." Image and Signal Processing and Analysis (ISPA), 2011 7th International Symposium on. IEEE, 2011.

[17] Tomar, Ranjeet Singh, et al. "Analysis of breast cancer using image processing techniques." Computer Modeling and Simulation, 2009. EMS'09 Third U KSim European Symposium on IEEE 2009.

[18] Green, Adele, et al. "Computer image analysis in the diagnosis of melanoma." Journal of the American Academy of Dermatology 31.6 (2010): 958-964.

[19] Nagaoka, T., et al. "Melanoma screening system using hyper spectral imager attached to imaging fiberscope." Engineering in Medicine and Biology Society (EMBC), 2012 Annual International Conference of the IEEE, 2012.

[20] Abuzaghleh, Omar, Buket D. Barkana, and Miad Faezipour. "SKINcure: A real time image analysis system to aid in the malignant melanoma prevention and early detection." Image Analysis and Interpretation (SSIAI) Southwest Symposium on IEEE 2014. 